\title{
Fluid-particle hybrid simulation on the transports of plasma, recycling neutrals, and carbon impurities in the Korea Superconducting Tokamak Advanced Research divertor region
}

\author{
Deok-Kyu Kim ${ }^{\text {a) }}$ and Sang Hee Hong ${ }^{\text {b) }}$ \\ Department of Nuclear Engineering, Seoul National University, Shinlim-dong, Gwanak-gu, \\ Seoul 151-742, Korea
}

(Received 2 August 2004; accepted 22 March 2005; published online 26 May 2005)

\begin{abstract}
A two-dimensional simulation modeling that has been performed in a self-consistent way for analysis on the fully coupled transports of plasma, recycling neutrals, and intrinsic carbon impurities in the divertor domain of tokamaks is presented. The numerical model coupling the three major species transports in the tokamak edge is based on a fluid-particle hybrid approach where the plasma is described as a single magnetohydrodynamic fluid while the neutrals and impurities are treated as kinetic particles using the Monte Carlo technique. This simulation code is applied to the KSTAR (Korea Superconducting Tokamak Advanced Research) tokamak [G. S. Lee, J. Kim, S. M. Hwang et al., Nucl. Fusion 40, 575 (2000)] to calculate the peak heat flux on the divertor plate and to explore the divertor plasma behavior depending on the upstream conditions in its base line operation mode for various values of input heating power and separatrix plasma density. The numerical modeling for the KSTAR tokamak shows that its full-powered operation is subject to the peak heat loads on the divertor plate exceeding an engineering limit, and reveals that the recycling zone is formed in front of the divertor by increasing plasma density and by reducing power flow into the scrape-off layer. Compared with other researchers' work, the present hybrid simulation more rigorously reproduces severe electron pressure losses along field lines by the presence of recycling zone accounting for the transitions between the sheath limited and the detached divertor regimes. The substantial profile changes in carbon impurity population and ionic composition also represent the key features of this divertor regime transition. (c) 2005 American Institute of Physics.
\end{abstract} [DOI: 10.1063/1.1914807]

\section{INTRODUCTION}

The introduction of divertor systems into tokamak fusion devices has enabled the advanced reactor operations for improving their confinement capability to control the global particle and energy balances. ${ }^{1,2}$ However, the excessive heat flux on the target divertor plate resulting from the increased plasma heating power level has become a crucial issue giving rise to severe restrictions on the reactor operation as confronted in many recent tokamak devices. For optimal design and operation of a tokamak reactor, it is important to accurately predict the peak heat load on the target divertor plate based on adequate understanding of the transport physics in the tokamak edge region. The edge transport physics, however, happens to be very complex due to the strong interactions among multiple particle species, e.g., plasma species (ions and electrons), recycling neutrals, and heavy element impurities, in the presence of the vessel wall and divertor structures.

In past decades, a number of edge plasma transport modelings have been attempted with success on the basis of fluid approaches. $^{3-5}$ On the other hand, the neutral particle transports were computed generally using large-scaled Monte Carlo codes with substantial treatment of kinetic effects. ${ }^{6-8}$

\footnotetext{
${ }^{a)}$ Present address: Agency for Defense Development, P.O. Box 35-5, Yuseong, Daejeon 305-600, Korea. Electronic mail: dkyukim@add.re.kr

${ }^{b)}$ Electronic mail: hongsh@snu.ac.kr
}

Some research groups also developed impurity transport codes based on the Monte Carlo scheme originally suggested by Stangeby. ${ }^{9-11}$ While significant numerical efforts have been made to perform edge modeling by combining these existing simulation codes for individual particle species in various tokamak applications, ${ }^{12-17}$ rarely found are the fully self-consistent divertor modelings, which are focused on elaborative couplings between a fluid plasma model and kinetic neutral/impurity models to demonstrate the characteristic evolution process of a recycling zone near the target divertor plate. Such limited numerical work done is mainly due to theoretical and numerical difficulties arising from the simultaneous consideration of multiple particle species in complex device geometries of tokamak machines. Recently, there was a numerical study that presented an intensive investigation of the detachment physics using a onedimensional simulation code incorporated with a neutral transport model that was simply tailored to match the Monte Carlo results or experimental data. ${ }^{18}$

In the present numerical work, we aim to present a fully coupled fluid-kinetic hybrid modeling for plasma, neutral, and impurity particle transports in a two-dimensional domain with the capability of demonstrating the formation progress of a recycling zone. The resultant simulation code enables us to account for the detachment phenomena more rigorously while most existing numerical codes still appear to have certain limitations in such applications. As numerical illustra- 
tions using the present simulation code, we perform the edge simulation of the Korea Superconducting Tokamak Advanced Research (KSTAR) tokamak, ${ }^{19}$ which is under construction at the Korea Basic Science Institute in Daejeon, Korea, in order to predict the physical characteristics of its divertor system when the coupled transports of plasma, recycling neutrals, and carbon impurities are taken into account simultaneously in the scrape-off layer (SOL). The integrated transport model describes the edge plasma as a single magnetohydrodynamic (MHD) fluid while treating the recycling neutrals and carbon impurities as kinetic particles based on the Monte Carlo techniques in a practically reduced geometry. Major physical interactions such as the collisions among particle species and between plasma and wall material are included utilizing relevant empirical or theoretical formulae.

In addition, there are great concerns about the anticipated high heat load and erosion rate on the divertor plate of KSTAR tokamak, since its power density is designed to reach as high as $1 \mathrm{MW} / \mathrm{m}^{3}$. This will bring about a critical problem in determination of the operation limit and the amount of heat removal required for the KSTAR divertor. Therefore, we also aim to estimate the peak heat and particle fluxes on the divertor plate at a normal incident angle and, furthermore, to explore the divertor plasma behavior depending on the upstream conditions in the base line operation mode of KSTAR tokamak so that we can obtain its reference operating range. Here, the upstream conditions are characterized by two key variables: input heating power and core boundary (separatrix) density. The calculated results from our fully coupled hybrid modeling are compared with those from the UEDGE modeling. ${ }^{20}$

\section{PHYSICAL MODELS AND NUMERICAL SCHEMES}

\section{A. Plasma transport}

Figure 1 shows a schematic diagram of the KSTAR tokamak cross section along with a computational grid mesh of the SOL. This orthogonal mesh, generated from MHD equilibrium calculations using the EFIT code, ${ }^{21}$ consists of 36 grids in the poloidal coordinate and 10 in the cross-field coordinate to reflect the magnetic configuration in the outer lower quadrant of the KSTAR SOL region for double-null operations. The alignment of the target divertor plate in this grid mesh structure corresponds to a zero tilt angle, i.e., the normal incidence of magnetic flux.

The core plasma moves outwards into the SOL region by cross-field diffusions across the last closed flux surface (LCFS) or the separatrix, and then it flows in both parallel and perpendicular directions to eventually reach the target divertor plate, on which the recycling and sputtering processes take place. During the transport process of plasma within the SOL channel, the initial temperature difference between ion and electron is appreciably reduced to a less significant level as a result of strong thermal relaxations. Thus, the single fluid MHD model has been commonly employed to describe the plasma transport in the SOL region with the assumption of vanishing current and thermal equilibration between ion and electron. ${ }^{22}$

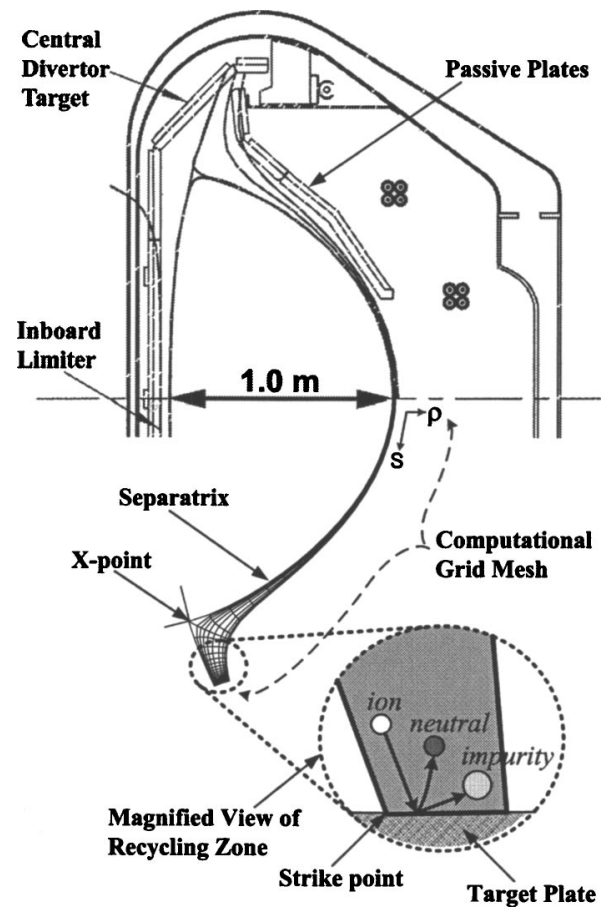

FIG. 1. Schematic diagram of the KSTAR tokamak cross section with the computational grid mesh illustrating the transports of plasma, recycling neutrals, and impurities within the recycling zone; $s$ and $\rho$ denote the poloidal and cross-field coordinates, respectively.

In this plasma transport modeling, the parallel transport is assumed to be classical with the flux-limited electron heat conduction taken into account, whereas the cross-field transport purely diffusive. The governing equations consist of the continuity, parallel momentum conservation, cross-field diffusion, and electron energy conservation equations in a steady state condition. These equations in a poloidal flux coordinate system $(s, \rho)$ of axisymmetric toroial configuration, where $s$ and $\rho$ denote the poloidal and cross-field coordinates, respectively, are written as follows:

(a) continuity equation

$$
\frac{1}{J} \frac{\partial}{\partial s}\left(\frac{J}{h_{s}} n u\right)+\frac{1}{J} \frac{\partial}{\partial \rho}\left(\frac{J}{h_{\rho}} n v\right)=S_{n},
$$

(b) parallel momentum conservation equation

$$
\begin{aligned}
\frac{1}{J} \frac{\partial}{\partial s} & \left(\frac{J}{h_{s}} n m u u_{\|}-\frac{J}{h_{s}^{2}} \mu_{s} \frac{\partial u_{\|}}{\partial s}\right) \\
& +\frac{1}{J} \frac{\partial}{\partial \rho}\left(\frac{J}{h_{\rho}} n m v u_{\|}-\frac{J}{h_{\rho}^{2}} \mu_{\rho} \frac{\partial u_{\|}}{\partial \rho}\right) \\
& =-\frac{B_{\theta}}{B} \frac{1}{h_{s}} \frac{\partial p}{\partial s}+S_{u_{\|}}
\end{aligned}
$$

(c) cross-field diffusion equation

$$
n v=-\frac{D_{\perp}}{h_{\rho}} \frac{\partial n}{\partial \rho},
$$

(d) electron energy conservation equation 


$$
\begin{aligned}
\frac{1}{J} \frac{\partial}{\partial s} & \left(\frac{J}{h_{s}} \frac{5}{2} n u T_{e}-\frac{J}{h_{s}^{2}} \kappa_{s} \frac{\partial T_{e}}{\partial s}\right) \\
& +\frac{1}{J} \frac{\partial}{\partial \rho}\left(\frac{J}{h_{\rho}} \frac{5}{2} n v T_{e}-\frac{J}{h_{\rho}^{2}} \kappa_{\rho} \frac{\partial T_{e}}{\partial \rho}\right) \\
& =\frac{u}{h_{s}} \frac{\partial p_{e}}{\partial s}+\frac{v}{h_{\rho}} \frac{\partial p_{e}}{\partial \rho}+S_{E} .
\end{aligned}
$$

In the above equations, $J, h_{s}$, and $h_{\rho}$ are the Jacobian and the scale factors of covariant vectors in the poloidal and crossfield directions, respectively; $m$ the deuterium ion mass; $B_{\theta} / B$ the magnetic field pitch; $n$ the plasma density in quasineutrality; $u$ and $u_{\|}$the poloidal and parallel fluid velocities, respectively, defined by $u=\left(B_{\theta} / B\right) u_{\|} ; v$ the crossfield diffusion velocity; $p$ and $p_{e}$ the total and electron partial pressures, respectively; $T_{e}$ the electron temperature; $\mu_{s}$ and $\mu_{\rho}$ the ion viscosities, and $\kappa_{s}$ and $\kappa_{\rho}$ the electron heat conductivities in the corresponding directions; $D_{\perp}$ the anomalous cross-field diffusion coefficient; and $S_{n}, S_{\mu_{\|}}$, and $S_{E}$ the volume sources of plasma particle, parallel momentum, and electron energy, respectively.

The poloidal components of ion viscosity and electron heat conductivity are obtained from the relations $\mu_{s}$ $=\left(B_{\theta} / B\right)^{2} \mu_{\|}$and $\kappa_{s}=\left(B_{\theta} / B\right)^{2} \kappa_{\|}$, respectively, where $\mu_{\|}$and $\kappa_{\|}$ are the parallel values given by the classical theory with the flux limited approximation. ${ }^{3,22}$ The cross-field transport coefficients $\mu_{\rho}$ and $\kappa_{\rho}$ are assumed anomalous and given by $\mu_{\rho}=D_{\perp} n m$ and $\kappa_{\rho}=\chi_{\perp}^{e} n$, where $D_{\perp}$ and $\chi_{\perp}^{e}$ are the plasma diffusion coefficient and electron heat diffusivity, respectively.

A set of conventional boundary conditions are applied to the modeling equations:

(a) on the midplane, a symmetry condition for plasma density and temperature with a flow stagnation,

$$
\frac{\partial n}{\partial s}=\frac{\partial T_{e}}{\partial s}=u=0,
$$

(b) on the outermost boundary and on the flux line between the $X$ point and the strike points, vanishing values of second derivatives for all variables in the cross-field direction,

$$
\frac{\partial^{2} u}{\partial \rho^{2}}=\frac{\partial^{2} n}{\partial \rho^{2}}=\frac{\partial^{2} T_{e}}{\partial \rho^{2}}=0,
$$

(c) on the divertor surface, the Bohm sheath criterion,

$$
u_{\|}=\sqrt{\frac{p}{n m}}, \quad q_{e \|}=\delta_{e} n u_{\|} T_{e},
$$

where $q_{e \|}$ is the electron's parallel heat flux into the sheath layer and $\delta_{e}$ the sheath energy transmission coefficient of electron which is approximated to be 4.5 in the present calculation, and

(d) on the LCFS, a uniformly distributed cross-field heat flux and a fixed plasma density,

$$
q_{e \perp}=\frac{f_{e} f_{\mathrm{down}} f_{\mathrm{out}}\left(1-f_{\mathrm{rad}}\right)}{A} P_{h}, \quad n=n_{s},
$$

where $q_{e \perp}$ is the electron's cross-field heat flux; $f_{e}$ the fraction of the heat transferred by electrons; $f_{\text {down }}$ and $f_{\text {out }}$ the fractions of the heat flowing in the directions downward and outward, respectively; $f_{\text {rad }}$ the fraction of the radiation energy loss within the core plasma; $A$ the LCFS area pertaining to the outer lower quadrant; $P_{h}$ the total input heating power; and $n_{s}$ the plasma density on the LCFS.

In the present modeling, the source terms in the Eqs. (1), (2), and (4) are written as

$$
\begin{aligned}
S_{n}= & n n_{0}\langle\sigma v\rangle_{\mathrm{ie}}-n n\langle\sigma v\rangle_{\mathrm{rec}}, \\
S_{u_{\|}}= & -\left(n n_{0}\langle\sigma v\rangle_{\mathrm{cx}}+n n\langle\sigma v\rangle_{\mathrm{rec}}\right) u_{\|}, \\
S_{E}= & -E_{\mathrm{ion}} n n_{0}\langle\sigma v\rangle_{\mathrm{ie}}-E_{\mathrm{ex}} n n_{0}\langle\sigma v\rangle_{\mathrm{ex}}-T_{e} n n\langle\sigma v\rangle_{\mathrm{rec}} \\
& -n n_{C}\left\langle L_{C}\left(T_{e}\right)\right\rangle,
\end{aligned}
$$

where $n_{0}$ is the neutral density; $\langle\sigma v\rangle_{\mathrm{ie}},\langle\sigma v\rangle_{\mathrm{ex}}$, and $\langle\sigma v\rangle_{\mathrm{cx}}$ are the reaction rate coefficients of deuterium atom for the electron impact ionization, electron impact excitation, and charge exchange, respectively; $\langle\sigma v\rangle_{\mathrm{rec}}$ is the rate coefficient of radiative recombination of deuterium ion. All the reaction rate coefficients are given as functions of either electron or ion temperature. ${ }^{1,23}$ The constants $E_{\mathrm{ion}}$ and $E_{\mathrm{ex}}$ denote the ionization potential and the excitation energy of deuterium atom, respectively; $n_{C}$ the carbon density; and $\left\langle L_{C}\left(T_{e}\right)\right\rangle$ the radiation cooling rate coefficient of carbon species averaged over all ionization stages in an equilibrium state. ${ }^{24}$

The plasma fluid equations subject to the relevant boundary conditions are discretized in a staggered grid structure using the finite volume method ${ }^{25}$ and the resulting pentadiagonal matrices are solved by a modified strongly implicit procedure. Strong underrelaxations are imposed on the iterations between the discretized fluid equations.

\section{B. Recycling neutrals and intrinsic carbon impurities}

The recycling neutrals are introduced in either atomic or molecular form depending on the incident energy of plasma ions to the divertor plate. Considering the short dissociation length of a deuterium molecule under typical edge conditions, it is sufficient to take account of only the atomic neutral sources: (a) Franck-Condon atoms and (b) directly reflected atoms. ${ }^{1,6,8}$ Neutral atoms can move toward the upstream region without being influenced by the magnetic fields but undergo interactions with background plasma: electron impact ionization, electron impact excitation, and charge exchange. In this recycling neutral simulation work, in order to describe the neutral motions and relevant physical interactions, we employ a Monte Carlo technique based on the suppressed absorption algorithm. ${ }^{6}$ The wall recycling and reflections are simulated using semiempirical formulas. ${ }^{26}$

Carbon or carbon composite is one of the best options for target materials owing to its high heat resistance and low-Z value, which is also the case of KSTAR tokamak. Though not clearly understood yet, it is believed that the physical sputtering of plasma ions dominates the production 
TABLE I. Design parameters of KSTAR tokamak for a base line operation mode.

\begin{tabular}{lc}
\hline \hline Parameters & Values \\
\hline Toroidal field & $3.2 \mathrm{~T}$ \\
Plasma current & $2 \mathrm{MA}$ \\
Major radius & $1.8 \mathrm{~m}$ \\
Minor radius & $0.5 \mathrm{~m}$ \\
Elongation at separatrix & 2 \\
Triangularity at separatrix & 0.8 \\
Pulse length & $20 \mathrm{sec}$ \\
Total auxiliary heating & $15.5 \mathrm{MW}$ \\
Plasma volume & $\sim 16 \mathrm{~m}^{3}$ \\
Plasma surface area & $\sim 48 \mathrm{~m}^{2}$ \\
Safety factor & $3-5$ \\
\hline \hline
\end{tabular}

of carbon impurities on the target divertor plate under the condition of high particle flux. ${ }^{2}$ Here, we utilize the physical sputtering yield function given by an analytic model ${ }^{27}$ to describe the carbon source. The transport of carbon impurity is more complicated than the deuterium recycling because it involves multiple ionization stages up to the fully stripped $\mathrm{C}^{6+}$. We adopt the numerical model for impurity transport given by Stangeby et al., ${ }^{9}$ which is also based on the Monte Carlo method, to trace the carbon particle in the SOL region. In each flight step of a carbon test particle, its charge state is determined from statistical calculations using the probability functions of ionization and recombination processes. ${ }^{23}$ The parallel motion of a single carbon particle is governed by the force balance between the frictional and thermal gradient forces as described in the following: $:^{9,22}$

$$
\begin{aligned}
m_{C} \frac{d v_{C \|}}{d t}= & F_{\text {friction }}^{C}+F_{\text {thermal }}^{C}=\frac{m u_{\|}-m_{C} v_{C \|}}{\tau_{s l}}+\alpha_{C} \nabla_{\|} T_{e} \\
& +\beta_{C} \nabla_{\|} T_{i},
\end{aligned}
$$

where $m_{C}$ is the carbon mass; $v_{C \|}$ is the parallel velocity of carbon; and $\tau_{s l}, \alpha_{C}$, and $\beta_{C}$ are the slowing down time, the coefficients of thermal gradient forces for ion and electron temperatures, respectively. In addition, we consider the random diffusive motions of carbon ions in both parallel and cross-field directions along with their thermalization processes due to collisions with background plasma. As de-
TABLE II. Input parameters used for KSTAR divertor simulation.

\begin{tabular}{lc}
\hline \hline Parameters & Values \\
\hline Heating power, $P_{h}$ & $1.5,8.0,15.5 \mathrm{MW}$ \\
Core boundary density, $n_{s}$ & $3,4,5 \times 10^{19} \mathrm{~m}^{-3}$ \\
Radiation loss fraction within the core plasma, $f_{\text {rad }}$ & $40 \%$ \\
Outboard/inboard power split & $3 / 1$ \\
Anomalous cross-field diffusion coefficient for plasma & $0.5 \mathrm{~m}^{2} / \mathrm{sec}$ \\
and impurity, $D_{\perp}$ & $1.0 \mathrm{~m}^{2} / \mathrm{sec}$ \\
Anomalous cross-field electron thermal diffusivity, $\chi_{\perp}^{e}$ & 0.12 \\
Flux limiting factor & 1.0 \\
Recycling ratio & \\
\hline \hline
\end{tabular}

scribed in the plasma transport model, the ion and electron temperature are assumed identical.

Transports of the recycling neutrals and carbon impurities are computed in a realistic geometry with the baffle gap, through which particles can escape out of the inner vessel container.

\section{SIMULATION RESULTS AND DISCUSSION}

\section{A. Input parameters}

Major design parameters of the KSTAR tokamak planned for its baseline operation mode are listed in Table I. The values of the input operating parameters are chosen to be compatible with the KSTAR fundamental phase. ${ }^{19}$ The total auxiliary heating power of $15.5 \mathrm{MW}$ is supplied by $8 \mathrm{MW}$ of neutral beam injection, $6 \mathrm{MW}$ ion cyclotron resonance heating, and 1.5 MW lower hybrid current drive. The core boundary density will be kept in the range of (3-5) $\times 10^{19} \mathrm{~m}^{-3}$ with the central plasma density reaching $\sim 1.0$ $\times 10^{20} \mathrm{~m}^{-3}$ below the density limit. ${ }^{28}$

A series of code simulations have been performed for different upstream conditions, which are parametrized by the input heating power $P_{h}$ and the separatrix (LCFS) plasma density $n_{s}$. It is assumed that the tokamak has the up-down symmetry for power division $\left(f_{\text {down }}=0.5\right)$ and the equal heat transfer capabilities for ion and electron $\left(f_{e}=0.5\right)$. Other input parameter values used for the simulations are shown in Table II. It is noted that, with a fixed value of the radiation loss fraction $f_{\text {rad }}$, the amount of plasma power entering the SOL region is directly proportional to the total input heating
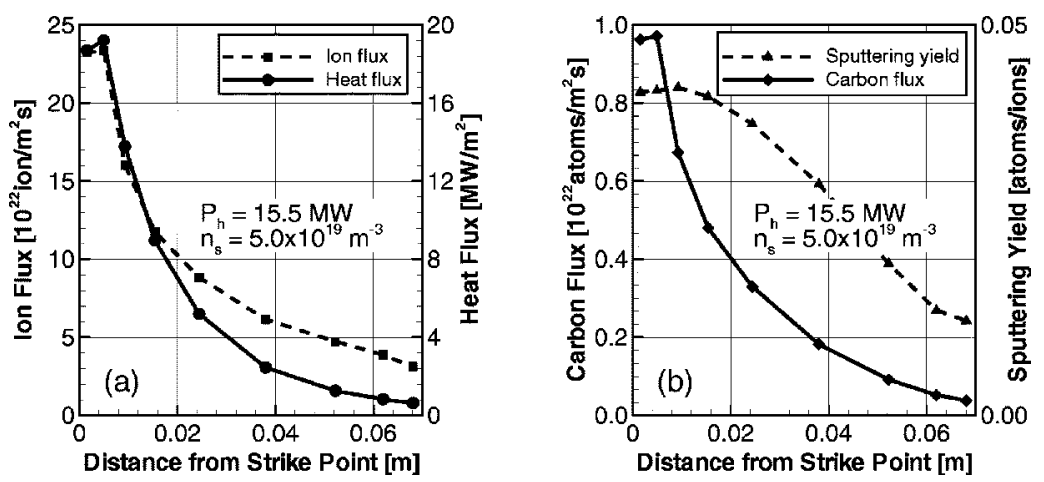

FIG. 2. Radial profiles of (a) the plasma heat and ion fluxes onto the target divertor plate and (b) the physical sputtering yield and particle flux of carbon impurity from the target surface for the KSTAR tokamak operated with $P_{h}=15.5 \mathrm{MW}$ and $n_{s}=5.0 \times 10^{19} \mathrm{~m}^{-3}$. 
power. However, the radiation loss fraction is usually found to be proportional to the average plasma density in tokamak experiments, ${ }^{2}$ and therefore the assumption of the fixed loss fraction value may result in uncertainties in estimating the heat flow rate to the target plate. In our numerical modeling, we simply use a relatively conservative value of radiative power loss fraction, $f_{\mathrm{rad}}=40 \%$, for the proposed base line operation mode of KSTAR machine. As a consequence, the power flow into the SOL region will be possibly overestimated at higher densities leading to a suppressed growth of the recycling zone in this simulation.

\section{B. Computed results}

\section{Heat and particle fluxes on the divertor in a full power operation}

The computed results by the present fluid-particle hybrid simulation code are shown in Fig. 2 for the radial (crossfield) profiles of (a) the heat and particle fluxes incident normal to the divertor plate and (b) the physical sputtering yield and the resultant carbon particle flux from the divertor surface when the upstream conditions of heating power $P_{h}$ $=15.5 \mathrm{MW}$ and the separatrix density $n_{s}=5 \times 10^{19} \mathrm{~m}^{-3}$ are given.

In Fig. 2(a), the peak heat flux reaches $18.7 \mathrm{MW} / \mathrm{m}^{2}$ near the strike point, which is about $10 \%$ higher than $\sim 17 \mathrm{MW} / \mathrm{m}^{2}$ obtained from the calculation by the existing UEDGE fluid code for the same divertor configuration. ${ }^{20}$ This discrepancy can be attributed to the use of a lower thermal diffusivity value as well as the fully two-dimensional kinetic treatments of recycling neutral particles by the Monte Carlo approach in the present divertor modeling, which may have resulted in a more peaked flux profile. At any rate, the computed results from both simulations simultaneously indicate that the peak heat flux at normal incident angle exceeds a tolerable engineering limit of $\sim 5 \mathrm{MW} / \mathrm{m}^{2}$ by a considerable amount at the full power heating condition. The UEDGE simulation previously indicated that the peak heat flux could be reduced by $50 \%$ at the target tilt angle of $\sim 45^{\circ}$. Even in this case, however, the still excessive heat flux needs to invite any active cooling techniques for safeguarded target protection. ${ }^{18,29-31}$

The target erosion rate estimated from Fig. 2(b) appears to be as high as $\sim 7.4 \mathrm{~mm} /$ day at the strike point of the graphite target plate. But, according to the approximation of equilibrium ionization balance, ${ }^{24}$ this amount of carbon flux

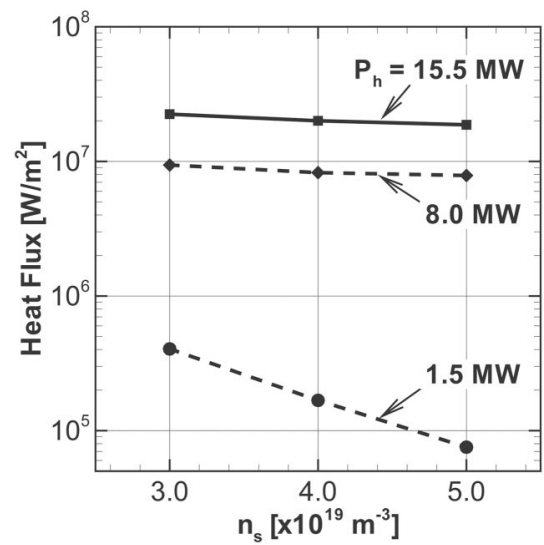

FIG. 3. Dependency of plasma heat flux at the strike point of the target divertor plate on input heating power $P_{h}$ and core boundary density $n_{s}$.

is far below the level at which the radiative cooling by carbon impurities significantly affects the energy transport in the SOL region.

\section{Correlations between upstream condition and divertor regime}

In order to examine the correlations between the upstream operating condition and the divertor regime, the similar calculations to those in the preceding section have been repeated to cover a wider operating range of heating power $P_{h}=1.5-15.5 \mathrm{MW}$ and separatrix density $n_{s}=(3-5)$ $\times 10^{19} \mathrm{~m}^{-3}$. The heat flux at the strike-point calculated for different heating powers are plotted in Fig. 3 as a function of separatrix density. The rapidly decreasing slope at $P_{h}$ $=1.5 \mathrm{MW}$ represents the detached divertor regime while the sheath-limited regimes appear at 8 and 15.5 MW with weak dependency of heat flux on density. Transitions from the sheath-limited to the detached divertor regime are frequently observed in practical situations where the power flow to the SOL region is reduced below a critical level or the plasma density is significantly increased. ${ }^{1,2}$

Such a transition of divertor regime seems closely linked to the presence of a neutral recycling zone. In cases where the recycling neutrals are allowed to penetrate deeper toward the upstream region, they can form a thick layer of cold neutrals in front of the target plate to further enhance the heat dispersal and momentum removal of the SOL plasma through plasma-neutral interactions.

The formation of a recycling zone is confirmed in Fig. 4,
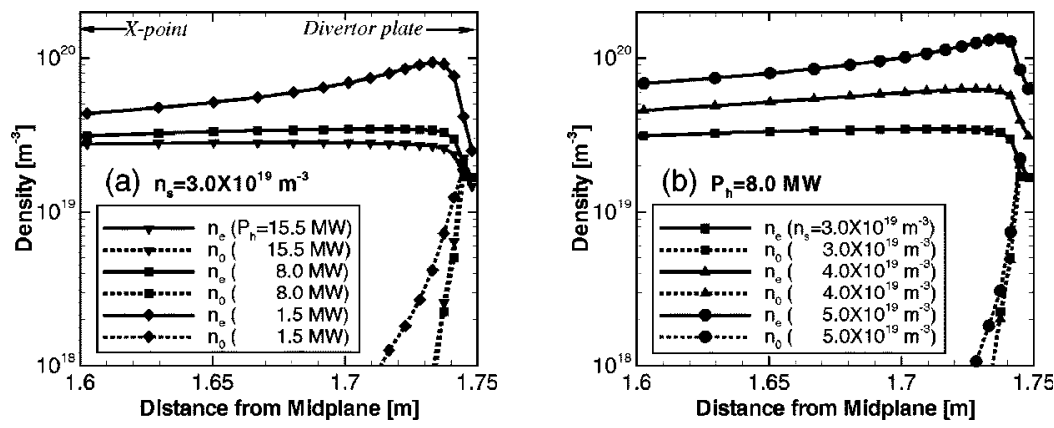

FIG. 4. Poloidal profiles of plasma and neutral densities along the separatrix line between the $X$ point and the divertor plate depending on (a) heating power $P_{h}$ and (b) core boundary density $n_{s}$. 
where the density profiles of plasma and recycling neutrals computed for different values of $P_{h}$ and $n_{s}$ are shown along the separatrix line between the $X$ point and the divertor plate. The calculations well demonstrate the early stage of divertor detachment by reducing the input heating power $P_{h}$ as seen in Fig. 4(a), and by increasing the plasma density $n_{s}$ in Fig. 4(b). ${ }^{18,29,30}$ The size of the recycling zone can be represented by the distance between the plasma density peak and the divertor plate, and it amounts to a few centimeters for the illustrated cases in Fig. 4. It should be noted that the average penetration depth of neutral atoms depends crucially on the temperature level in the divertor domain. This is because the ionization rate coefficient of deuterium atom drops rapidly as the plasma temperature decreases below $100 \mathrm{eV}$ whereas the charge-exchange rate coefficient is much less sensitive to the temperature. At sufficiently low temperature, therefore, the recycling neutral has a long ionization mean-free path with a higher survival probability. It then follows that the control of plasma power flow into the SOL region is of great importance in achieving the detachment or high-recycling conditions at the divertor. Moreover, according to a theoretical study, ${ }^{31}$ the development of recycling zone is crucial to achieving a high edge cooling capacity with a larger amount of radiation losses allowed in the edge region.

\section{Pressure loss along the magnetic field line}

The presence of the recycling zone inevitably leads to the destruction of total (static+dynamic) pressure conservation along the magnetic field line due to intensive ion-neutral collisions. A measure of the parallel pressure drop provides a useful information on the effect of recycling zone and, furthermore, a good estimation of the degree of divertor detachment. There would be a larger amount of momentum losses as the recycling zone extends in size allowing more collisions to take place.

The parallel pressure loss through the recycling zone can be described by a simple expression derived from the parallel momentum balance equation

$$
\frac{\partial}{\partial s_{\|}}\left(n m u_{\|} u_{\|}+p\right)=S_{u_{\|}}
$$

where $s_{\|}$is the parallel length coordinate in the direction toward the divertor plate with its origin located at the midplane. The viscosity term is ignored for simplicity. The static pressure term $p$ includes both the ion and electron pressures. Integrating Eq. (13) from the midplane to the divertor surface using the Bohm sheath boundary condition yields

$$
2 p^{\mathrm{div}}-p^{\mathrm{mid}}=\int_{\text {mid }}^{\text {div }} S_{u_{\|}} d s_{\|},
$$

where $p^{\mathrm{div}}$ and $p^{\mathrm{mid}}$ are the static pressures at the divertor plate and the midplane, respectively. The pressure loss factor $f_{m}$ is defined as ${ }^{2}$

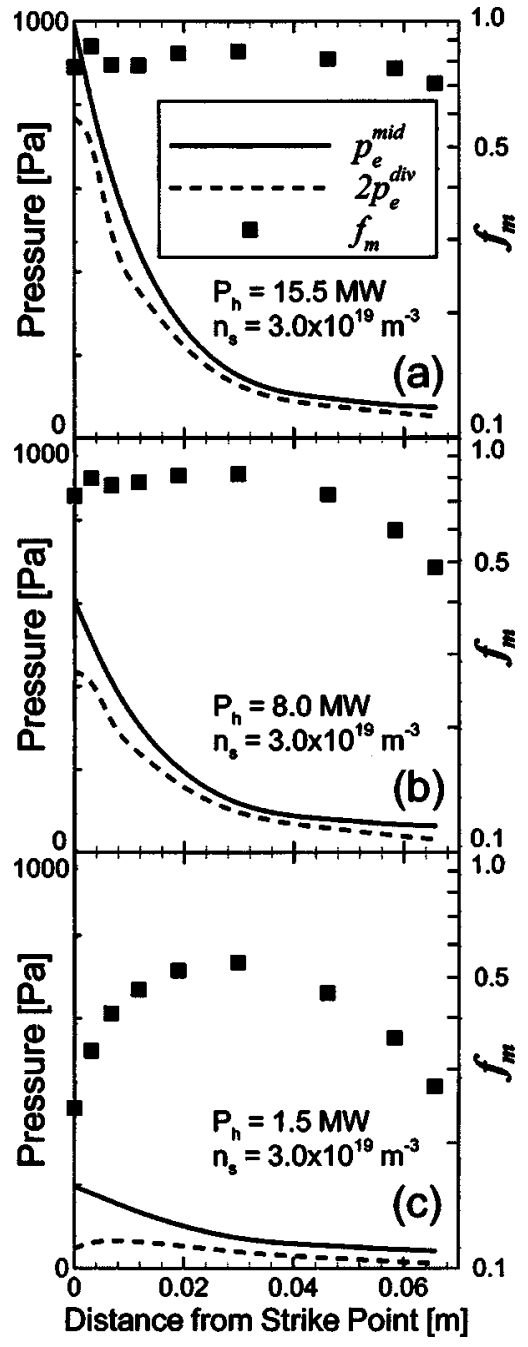

FIG. 5. Comparison of radial electron pressure profiles on the midplane and the divertor plate with the corresponding pressure loss factors $f_{m}$ computed for different heating powers of (a) 15.5, (b) 8.0, and (c) 1.5 MW with a fixed density of $n_{s}=3.0 \times 10^{19} \mathrm{~m}^{-3}$.

$$
f_{m} \equiv \frac{2 p^{\mathrm{div}}}{p^{\text {mid }}}=\frac{2 p_{e}^{\mathrm{div}}}{p_{e}^{\text {mid }}}=1+\frac{\int_{\mathrm{mid}}^{\mathrm{div}} S_{u_{\|}} d s_{\|}}{p^{\mathrm{mid}}} .
$$

It is supposed that all the momentum transfer processes occur within the recycling zone since the neutral density rapidly decreases toward the upstream region. Then, Eq. (15) reduces to

$$
f_{m} \cong 1+\left(\frac{B_{\theta}}{B}\right)^{-2} \frac{\left\langle S_{u}\right\rangle}{p^{\text {mid }}} \Delta,
$$

where $\left\langle S_{u}\right\rangle$ is the poloidal momentum source averaged over the recycling zone and $\Delta$ is the effective size of the recycling zone. The term $\left\langle S_{u}\right\rangle$ has a negative value because plasma delivers its momentum to the cold neutral particles through collisions in the recycling zone. Therefore, the pressure loss factor $f_{m}$ appears smaller than 1 indicating that the parallel plasma pressure is not conserved along the field line in the presence of the recycling zone.

Figure 5 compares the radial pressure profiles calculated on the midplane and the divertor plate to show their corre- 


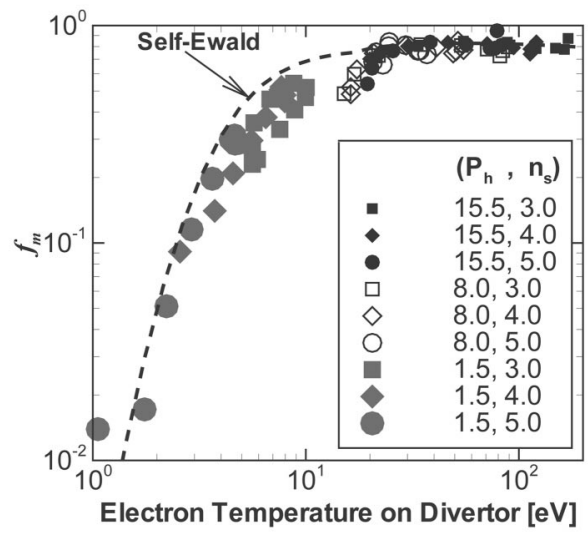

FIG. 6. Pressure loss factor $f_{m}$ vs electron temperature at the target divertor plate computed for various heating powers $P_{h}$ (MW) and separatrix densities $n_{s}\left(\times 10^{19} \mathrm{~m}^{-3}\right)$; the dashed curve is from an analytic two-point model given by Self and Ewald.

sponding $f_{m}$ values for different input powers with a fixed density of $n_{s}=3.0 \times 10^{19} \mathrm{~m}^{-3}$. Low values of $f_{m}$ are indicative of a well-developed recycling zone and a subsequent occurrence of detachment. It is noted that the detachment takes place narrowly on the outer wall side as seen in Fig. 5(b) for the 8.0 MW power case, but that it spreads out over the whole radial region especially with deep penetrations near the strike point and the wall side as observed in Fig. 5(c) for the lower power of 1.5 MW. Qualitatively similar detachment patterns are found in some experiments exhibiting their dependencies on target plate configurations and operating conditions. ${ }^{32}$

Figure 6 presents a plot of the pressure loss factor versus the target electron temperature in comparison with an analytic two-point model given by Self and Ewald. ${ }^{2,33}$ The analytic curve shows qualitative agreement with the results from our two-dimensional hybrid simulation but tends to underestimate the pressure drop at low temperatures less than $20 \mathrm{eV}$. We consider that this discrepancy is due to the inclusions of the cross-field diffusion and the parallel temperature gradient within the recycling zone in addition to the momentum transfer by charge-exchange interactions in the present fluidparticle simulation. ${ }^{34}$

\section{Relationship between cross-field transport coefficients and peak heat flux}

The cross-field radial profiles of plasma density and temperature in the SOL is mainly determined by the transports of plasma and energy across the magnetic flux surfaces, which are commonly described using anomalous cross-field transport coefficients, for example, plasma diffusion coefficient $D_{\perp}$ and electron thermal diffusivity $\chi_{\perp}^{e}$. Therefore, the relationship between the cross-field transport coefficients and the peak heat flux to the divertor plate is considered to be rather straightforward to predict in a qualitative manner. As the coefficient values of cross-field transports increase, the radial profile of heat flux on the divertor plate is expected to become broader and consequently the peak value of heat flux reduces, which appears to be more beneficial to the target material protection. Nevertheless, the cross-field transports

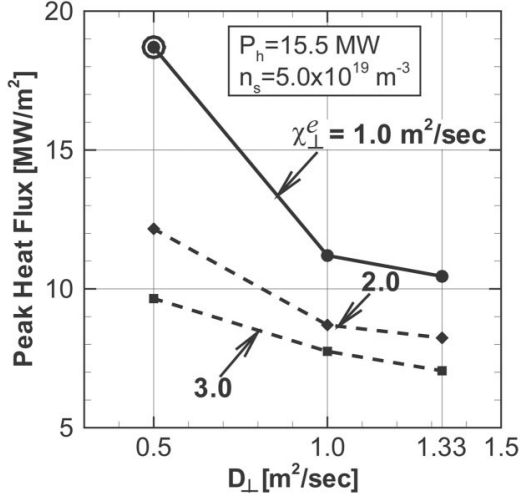

FIG. 7. Dependency of peak heat flux to the target divertor plate on the cross-field plasma diffusion coefficient $D_{\perp}$ and the cross-field electron thermal diffusivity $\chi_{\perp}^{e}$. The symbol ${ }_{-}$represents the reference modeling condition with $D_{\perp}=0.5 \mathrm{~m}^{2} / \mathrm{sec}$ and $\chi_{\perp}^{\bar{e}}=1.0 \mathrm{~m}^{2} / \mathrm{sec}$.

should be suppressed to ensure better confinements of plasma and energy for efficient tokamak operations.

It is of practical interest to examine the dependency of target peak heat flux on cross-field transport coefficients quantitatively. Figure 7 shows the peak heat fluxes computed for different values of the cross-field coefficients in the proposed KSTAR base line mode, $D_{\perp}=0.5 \sim 1.33 \mathrm{~m}^{2} / \mathrm{sec}$ and $\chi_{\perp}^{e}=1.0 \sim 4.0 \mathrm{~m}^{2} / \mathrm{sec}$ at $P_{h}=15.5 \mathrm{MW}$ and $n_{s}=5 \times 10^{19} \mathrm{~m}^{-3}$. From this figure, the peak heat flux appears at an acceptable level for the higher coefficient values of $D_{\perp}>1.0$ and $\chi_{\perp}^{e}$ $>2.0$. However, considering that the cross-field transports should be maintained substantially low in a tokamak, the power dispersion through detachment formation would be necessarily required for reducing the peak heat flux to the divertor plate.

\section{Transport of carbon impurities from physical sputtering source}

Figure 8 shows the poloidal density distributions of carbon ions, which are generated from the physical sputtering of divertor surface, along the separatrix line for heating powers of $P_{h}=$ (a) 15.5 and (b) $1.5 \mathrm{MW}$ with a fixed density of $n_{s}$ $=3.0 \times 10^{19} \mathrm{~m}^{-3}$. It is clearly seen that both the absolute level of total carbon density and the relative ionic compositions are also strongly dependent on the electron temperature level near the divertor plate. For the highest heating power condition, i.e., $P_{h}=15.5 \mathrm{MW}$ in Fig. 8(a), the total carbon density value reaches the same order of magnitude of recycling neutral density particularly near the target surface but rapidly decreases toward the upstream, and $\mathrm{C}^{4+}$ ions dominate the entire divertor region. Carbon ions with higher charge numbers, $\mathrm{C}^{5+}$ and $\mathrm{C}^{6+}$, are also seen in this full-powered operation mode. On the contrary, for the lowest heating power of $P_{h}=1.5 \mathrm{MW}$ in Fig. 8(b), the total carbon density is significantly reduced by more than two orders of magnitude, which is consistent with the physical consideration that the carbon yield by physical sputtering falls below $10^{-3}$ for the incident energy of deuterium ion less than $30 \mathrm{eV}$. Besides, near the divertor surface where the background plasma temperature is not sufficiently high in this low power regime, $\mathrm{C}^{4+}$ ions ap- 

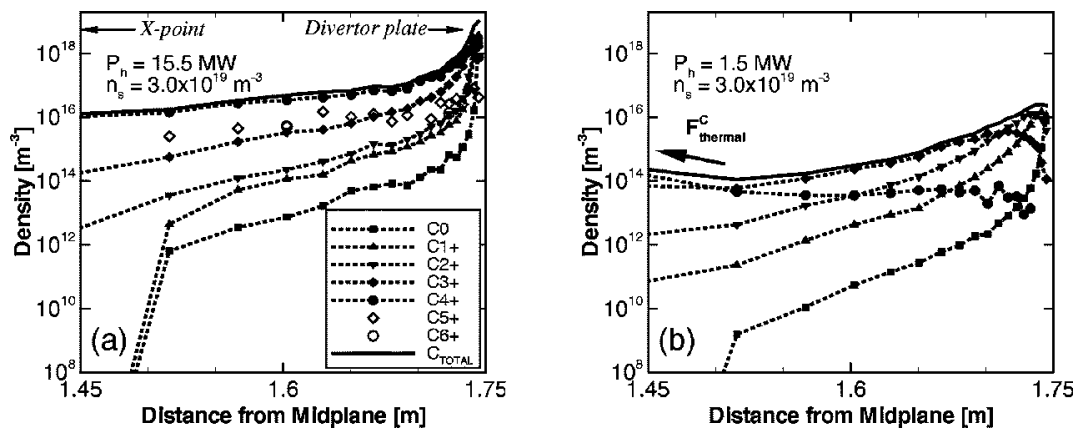

FIG. 8. Poloidal profiles of carbon ion densities sputtered from the target divertor plate for heating powers of (a) 15.5 and (b) $1.5 \mathrm{MW}$ with a fixed density of $n_{s}=3.0 \times 10^{19} \mathrm{~m}^{-3}$.

pear to be minor in population and both $\mathrm{C}^{5+}$ and $\mathrm{C}^{6+}$ ions are not even found in Fig. 8(b). Nevertheless, the $\mathrm{C}^{4+}$ ion density gradually rises along the field line toward the upstream to become dominant in the region away from the target divertor surface. Such density increase toward the $X$ point clearly reveals the effect of thermal gradient force $F_{\text {thermal }}^{C}$ that drives the carbon ions toward the higher temperature region as depicted in Fig. 8(b).

A more precise modeling of the carbon impurity transport in a tokamak device calls for clear understanding of the impurity production mechanism including chemical sputterings, and requires extensive radiation loss data for nonequilibrium states. ${ }^{35-38}$

\section{CONCLUSIONS}

A two-dimensional fluid-particle hybrid code has been developed for self-consistent simulation of the fully coupled transports of plasma, recycling neutrals, and carbon impurities in the tokamak divertor region, and used for calculations of the ion, carbon, and heat fluxes on the divertor plate and the plasma, neutral, and impurity ion densities in the SOL region in the KSTAR base line mode operated with input heating powers of $1.5-15.5 \mathrm{MW}$ and core boundary densities of $(3-5) \times 10^{19} \mathrm{~m}^{-3}$. The simulated results indicate that the peak heat flux on the KSTAR divertor plate may exceed the engineering limit in the full-powered operation of 15.5 MW, and that the target heat flux can be decreased to an acceptable level by the formation of extended recycling zone which is achievable by reducing the heating power flow into the SOL and by increasing the core boundary density. In addition, the correlation between the upstream operating condition and the divertor regime transition has been examined in this proposed range of KSTAR operating parameters. An extended recycling zone turns out to enhance the power dispersal in the divertor region and the pressure drop along the magnetic field line, and to eventually cause the divertor regime transition. The calculated results effectively demonstrate the early stage of divertor detachment in a similar fashion observed in some experiments with reduced heat flow into the SOL region, and clearly show a close physical connection of the detachment with the parallel pressure drop through the neutral recycling zone. Furthermore, the characteristic diagram of pressure loss factor versus electron temperature obtained from the present two-dimensional hybrid simulation shows qualitative agreement with the prediction made by a simple analytical two-point model but indicates that the analytic model underestimates pressure drops at low temperatures due to its one-dimensional limitation. The intrinsic carbon impurities generated by physical sputtering on the divertor plate exhibit distinct changes in their density and ionic composition profiles according to the SOL temperature level, which also characterizes the divertor regime. Despite some uncertainties included in the numerical model used for describing the edge transport phenomena, results from the present hybrid simulation can be of practical use for design and operation of diverted tokamaks owing to its fully coupled two-dimensional self-consistent features.

${ }^{1}$ P. C. Stangeby, Plasma Boundary of Magnetic Fusion Devices (Institute of Physics, Bristol, 2000); P. C. Stangeby and G. M. McCracken, Nucl. Fusion 30, 1225 (1990).

${ }^{2}$ C. S. Pitcher and P. C. Stangeby, Plasma Phys. Controlled Fusion 39, 779 (1997).

${ }^{3}$ B. J. Braams, Ph.D. thesis, University of Utrecht, Utrecht, The Netherlands, 1986.

${ }^{4}$ T. D. Rognlien, J. L. Milovich, M. E. Rensink, and G. D. Porter, J. Nucl. Mater. 196-198, 347 (1992).

${ }^{5}$ D. A. Knoll, P. R. McHugh, S. I. Krasheninnikov, and D. J. Sigmar, Phys. Plasmas 3, 293 (1996).

${ }^{6}$ D. B. Heifetz and D. E. Post, Comput. Phys. Commun. 29, 287 (1983).

${ }^{7}$ D. Reiter, Chr. May, D. Coster, and R. Schneider, J. Nucl. Mater. 220222, 987 (1995).

${ }^{8}$ D. K. Kim, S. H. Hong, and K. Im, J. Korean Nucl. Soc. 29, 459 (1997); in Current Topics in Physics, edited by Y. M. Cho, J. B. Hong, and C. N. Yang (World Scientific, Singapore, 1998), Vol. 2, p. 1110.

${ }^{9}$ P. C. Stangeby, C. Farrel, S. Hoskins, and L. Wood, Nucl. Fusion 28, 1945 (1988).

${ }^{10}$ K. Shimizu, H. Kubo, T. Takizuka et al., J. Nucl. Mater. 220-222, 410 (1995).

${ }^{11}$ See National Technical Information Service Document No. DE98005689 (General Atomics Report No. GA-A22831 by T. E. Evance and D. F. Finkelthal, 1998).

${ }^{12}$ D. P. Coster, R. Schneider, J. Neuhauser et al., J. Nucl. Mater. 241-243, 690 (1997).

${ }^{13}$ M. E. Fenstermacher, G. D. Porter, M. E. Rensink et al., J. Nucl. Mater. 220-222, 330 (1995).

${ }^{14}$ ITER Physics Expert Group on Divertor, ITER Physics Expert Group on Divertor Modelling and Database, and ITER Physics Basis Editors, Nucl. Fusion 39, 2391 (1999).

${ }^{15}$ K. Im, D. K. Kim, and S. H. Hong, J. Korean Phys. Soc. 29, 52 (1996); D. K. Kim, J. M. Ryu, S. H. Hong, and K. Im, ibid. 31, S223 (1997).

${ }^{16}$ D. K. Kim and S. H. Hong, in Proceedings of the First General Assembly of Asian Plasma and Fusion Association Joint with the Third Asia Pacific Plasma theory Conference, Chinese Physics Letters, Special Issue (Chinese Physical Society, Bejing, 1999), pp. 101-104.

${ }^{17}$ J. S. Ko, K. H. Im, D. K. Kim, and S. H. Hong, IEEE Trans. Plasma Sci. 30, 64 (2002); J. S. Ko, D. K. Kim, S. H. Hong, and K. Im, J. Korean Phys. Soc. 41, 212 (2002).

${ }^{18}$ R. Goswami, P. Caw, M. Warrier et al., Phys. Plasmas 8, 857 (2001).

${ }^{19}$ G. S. Lee, J. Kim, S. M. Hwang et al., Fusion Eng. Des. 46, 405 (1999); Nucl. Fusion 40, 575 (2000).

${ }^{20}$ B. J. Lee, D. Hill, K. H. Im et al., Fusion Technol. 37, 2000 (2000). 
${ }^{21}$ L. L. Lao, H. S. John, R. D. Stambaugh et al., Nucl. Fusion 25, 1611 (1985).

${ }^{22}$ S. I. Braginskii, in Reviews of Plasma Physics, edited by A. M. A. Leontovich (Consultants Bureau, New York, 1965), Vol. 1, p. 205.

${ }^{23}$ I. H. Hutchinson, Principles of Plasma Diagnostics (Cambridge University Press, Cambridge, 1987).

${ }^{24}$ D. E. Post, R. V. Jensen, C. B. Tarter et al., At. Data Nucl. Data Tables 20, 397 (1977)

${ }^{25}$ S. V. Patankar, Numerical Heat Transfer and Fluid Flow (Hemisphere, New York, 1980).

${ }^{26}$ W. Eckstein and H. Verbeek, in Data Compendium for Plasma Surface Interactions, Nuclear Fusion Special Issue, edited by R. A. Langley (International Atomic Energy Agency, Vienna, 1984), p. 12.

${ }^{27}$ J. Bohdansky, in Data Compendium for Plasma Surface Interactions, Nuclear Fusion Special Issue, edited by R. A. Langley (International Atomic Energy Agency, Vienna, 1984), p. 61.

${ }^{28}$ M. Greenwald, J. L. Terry, S. M. Wolfe et al., Nucl. Fusion 28, 2199 (1988).
${ }^{29}$ G. F. Mathews, J. Nucl. Mater. 220-222, 104 (1995).

${ }^{30}$ S. Nakazawa, N. Nakajima, M. Okamoto, and N. Ohyabu, Contrib. Plasma Phys. 40, 491 (2000).

${ }^{31}$ K. Borrass and G. Janeschitz, Nucl. Fusion 34, 1203 (1994).

${ }^{32}$ M. Keilhacker et al., Plasma Phys. Controlled Fusion 37, A3 (1995); B. LaBombard, J. Goetz, C. Kurz et al., Phys. Plasmas 2, 2242 (1995).

${ }^{33}$ S. A. Self and H. N. Ewald, Phys. Fluids 9, 2486 (1966).

${ }^{34}$ P. C. Stangeby, Nucl. Fusion 33, 1695 (1993).

${ }^{35}$ G. F. Mathews, P. C. Stangeby, J. D. Elder et al., J. Nucl. Mater. 196-198, 374 (1992).

${ }^{36}$ J. Roth and C. Garcia-Rosales, Nucl. Fusion 36, 1647 (1996).

${ }^{37}$ K. Shimizu, T. Takizuka, and A. Sakasai, J. Nucl. Mater. 241-243, 167 (1997).

${ }^{38}$ See National Technical Information Service Document No. DE98001785 (General Atomics Report No. GA-A22693 by T. E. Evance, D. F. Finkelthal, Y. S. Loh et al., 1997). 